\title{
Automated Stock Trading - Developing the Serious Game FSTG to Teach the Topic of Finite State Machines
}

\author{
https://doi.org/10.3991/ijep.v7i1.6524 \\ M.C. Utesch \\ Staatliche Fachober- und Berufsoberschule Technik München, Munich, Germany \\ Technical University of Munich (TUM), Munich, Germany \\ utesch@in.tum.de \\ A. Hauer \\ Technical University of Munich (TUM), Munich, Germany \\ a.hauer@tum.de \\ R. Heininger \\ Technical University of Munich (TUM), Munich, Germany \\ robert.heininger@in.tum.de \\ H. Krcmar \\ Technical University of Munich (TUM), Munich, Germany \\ krcmarein.tum.de
}

\begin{abstract}
A new methodology to teach about the topic of Finite State Machines is presented in this paper. For this purpose, nine learning objectives which are classified into the three categories Basics of Finite State Machines, parallels between Finite State Machines and stock trading, and the application of Finite State Machines were developed and implemented into a Serious Game solution. This paper covers the third category about the application of Finite State Machines and its use to create Artificial Intelligence. The learning objectives were determined by using the widely-known Taxonomy of Bloom and integrated into the Serious Game The Finite State Trading Game (FSTG). In this turn-based trading game, the user aims to beat a Non-Player Character by skillfully trading shares while the user faces increasing complexity throughout the game. For the evaluation of the Serious Game approach a pre-test and post-test setting was performed with students of a local upper vocational school class at the Technical University of Munich. The results of the following analysis demonstrated significant progress in terms of the students' knowledge about Finite State Machines for every tested statement.
\end{abstract}

Keywords-Artificial Intelligence, Bloom's taxonomy for learning objectives, Finite State Machine, Serious Game, stock trading 


\section{Introduction}

Computer games are enjoying an increasing popularity all over the world: for example, the average gamer plays twelve years in his or her life and 72 percent of the households in the United States playing video games [1]. But yet many people are not aware of the fact that all these games - as well as many other applications, like traffic lights - are based on so-called Finite State Machines (FSMs). FSMs are used to create and represent the logic of these actions. The National Institute for Standardization and Technology (NIST) defines an FSM as a "model of computation consisting of a set of states, a start state, an input alphabet, and a transition function that maps input symbols and current states to a next state" [2].

FSMs represent a core concept regarding automation. Automation is part of the curriculum at upper vocational school (UVS) in Bavaria, Germany [3], as well as at IT-related degree courses, e.g. in the Bachelor's Program of the Technical University of Munich (TUM) [4, 5].

The curriculum for 12th graders at UVS in Bavaria, Germany, [3] covers a special class dedicated to engineering, called 'Technology and Computer Science'. This class comprises the modules basics of modern programming languages, programming styles and data structures, and object-oriented programming. They are the basis for the next modules capstone project and systems and processes. The capstone project aims at understanding, analyzing and evaluating a complex technical system like a power station or a car, as well as developing and implementing problem-solving strategies which are especially suited for this kind of technical system. With systems and processes the students are expected to gain a better understanding of technical systems and processes. They should be able to identify the essential elements and processes of a technical system, and describe their contribution to its overall functionality. The students learn to understand and apply systems as well as to analyze and evaluate them [6].

Using the so-called State Pattern, an FSM representation can be developed for almost every IT-based application ranging from control systems to business simulations and even computer games [7]. These State Patterns are the optimum architecture for the correct implementation of an FSM. Because of the ubiquity of FSM in reality, teaching FSM to UVS students seems to be a promising and valuable approach to gain a better understanding of real world scenarios. Yet - which application would be best suited to meet the learning of UVS students? Traffic lights build on a small number of states and are present everywhere to control traffic in our daily life. A more challenging FSM application would be a stock trading system and its processes of buying and selling shares [6]. A share is traded if the offered share price is equal or lower than the limit price and a potential buyer has the money to invest in the share.

Using educational software is a common trend [8]. Serious Games promise to support self-regulated personalized learning (SRPL) [9]. Two examples shall clarify how playing Serious Games may encourage SRPL. As a first example, Utesch, et al. [10] describes a learning scenario for UVS students in the context of the Pupils' Academy of Serious Gaming. An important goal of the academy is to reduce the dropout rate of students by strengthening their study skills even before they start studying at universi- 
ty. The ERPsim ${ }^{T M}$ Distribution business game is used to improve activities related to e.g. time management or teamwork. Secondly, Andergassen, et al. [11] presents the learning platformLearn@WU aiming at better ways for self-learning. Furthermore, the trend towards Serious Gaming is supported by the increasing availability of computers in German classrooms. In the year 2011, already about $90 \%$ of the schools leading to the German Abitur provided access to Personal Computers for the students [12]. Thus, summarizing the previous considerations, a Serious Game approach based on an FSM example seems to be well suited for learning about systems and processes in 'Technology and Computer Sciences' classes in Bavarian UVS.

The UVS students are young people who have realized at secondary school level: "I learn best in an application-oriented way" [10, 13]. Therefore, we developed a stock trading game named 'The Finite State Trading Game' (FSTG) [6]. With FSTG the learners strive to skillfully trade shares in order to beat a Non-Player Character (NPC) through higher profits. The scenario of automated stock trading was used for two reasons: Firstly, stock trading is a challenging FSM ex-ample out of everyday life. Secondly, automated stock trading is strongly related to the science of Information Systems. As a precondition, the IT-related knowledge is very heterogeneous at UVS due to a large variety of the students' learning histories. Thus, the proposed Serious Game solution should address FSM from scratch. This leads to the following research question [6]:

How to design, create, and present a Serious Game to teach the topic of Finite State Machines to upper vocational school students in Bavaria using stock trading as an example?

This research question was solved by four steps [6].

- In the first step we reviewed literature to identify the most important learning objectives. Additionally, we classified and verified them using the Taxonomy of Bloom [14] in the revision of Krathwohl [15] (chapter 2).

- In a second step, we integrated these learning objectives into a Serious Game solution (chapter 3).

- In the third step, the software was tested with UVS students. The students' performance was measured by a pre-test and post-test scenario.

- In the fourth step we evaluated the software. The students' learning progress was quantified and analyzed in order to prove the suitability of the Serious Game solution for the target group (chapter 4).

\section{The Learning Objectives}

For being able to teach students about FSMs, the first essential step is to describe the components of an FSM. According to Yuan and Qu [16] an FSM "consists of a finite set of states, a start state, an input alphabet, and a transition function that defines the next state based on the current state and input symbols." It is important to note, that the denomination of the elements of an FSM is not standardized. While the 
term state is used in practically every literature due to its occurrence in the term Finite State Machine, the other elements appear in different names in the literature.

In order to teach the concept of FSMs, three steps A), B) and C) have to be conducted.

a) The basic elements and properties of an FSM are introduced to the students, in combination with the most common representation possibilities [6].

b) By using stock trading as an example, the students should gain knowledge about a real world FSM application and its parallels to automation.

c) The students should analyze the application of FSMs to construct an Artificial Intelligence as well as evaluate its benefits and limitations.

Consequently, we developed nine learning objectives for these steps by analyzing and evaluating a couple of representative FSM definitions and applications [6]. The learning objectives are equally distributed among the three steps.

A common way to structure learning objectives is to describe them according to Bloom's Taxonomy (see Fig. 1). Thus, in order to create, classify, and verify the learning objectives, we relied on this Taxonomy of educational objectives [14]. The relevant aspects of this taxonomy will be shortly summarized in the following, for a better understanding of the FSTG approach

Bloom's Taxonomy started as a one-dimensional structure providing the six categories Knowledge, Comprehension, Application, Analysis, Synthesis, and Evaluation [14]. In the year 2001 a revision of the original taxonomy was published by Lorin W. Anderson and David R. Krathwohl [17]. It transformed the one-dimensional structure into a two-dimensional matrix. The vertical dimension is called the Knowledge Dimension, while the horizontal dimension is entitled the Cognitive Process Dimension. Since "statements of objectives typically consist of a noun [...] - the subject matter content - and a verb [...] - the cognitive process(es)" [15], the two-dimensional table eases to distinguish the subject matter content and the respective cognitive process, when classifying a learning objective [15]. Since the first version of the taxonomy has been provided by Bloom, also the two-dimensional matrix of the taxonomy will furthermore be addressed as Bloom's Taxonomy in this paper.

The Knowledge Dimension describes the type of knowledge the learning objective approaches. The Factual Knowledge contains learning objectives dealing with "basic elements that students must know to be acquainted with a discipline or solve problems in it" [15]. So this row offers a classification option for learning objectives covering elementary terms and principles. The Conceptual Knowledge is described in Krathwohl's paper as "The interrelationships among the basic elements within a larger structure that enable them to function together" [15]. This means that assembling the terms and principles that students have learned in the Factual Knowledge to a coherent structure approaches the Conceptual Knowledge. Furthermore the Procedural Knowledge is specified as "methods of inquiry, and criteria for using skills, algorithms, techniques, and methods" [15]. Thus, Procedural Knowledge covers learning objectives handling sequential processes. The last knowledge type is called Metacognitive Knowledge. Being described as "Knowledge of cognition in general as well as 
awareness and knowledge of one's own cognition" [15], learning objectives classified into this row deal with the student's way of thinking and producing content.

The second dimension of the taxonomy is called Cognitive Process Dimension. This dimension classifies how the students shall approach the knowledge. The terms used for the column names are examples for the actual words to be used when phrasing the learning objectives, and are thus in a verb form. The first column is called Remember. Krathwohl [15] describes this process as "retrieving relevant knowledge from long-term memory". So this cognitive process addresses learning objectives dealing with repeating learned content. The second column of the dimension, which is named Understand, shall contain learning objectives about "determining the meaning of instructional messages" [15]. Such learning objectives deal with grasping the gist of the transferred content and recognizing its elementary components. In the column Apply the focus of the learning objectives shifts from learning by reading and hearing to learning by doing, formulated as "Carrying out or using a procedure in a given situation" [15]. The next column - called Analyze - is used for the classification of learning objectives which are about "breaking material into its constituent parts and detecting how the parts relate to one another and to an overall structure or purpose" [15]. Decomposing a structure, process, or concept into its elements and inspecting them helps the students to understand the composition as a whole. The fifth column is called Evaluate. Like the name says, learning objectives in this column deal with evaluating content properly to get to know the content, or like Krathwohl describes in his revision as "making judgments based on criteria and standards" [15]. The last column is named Create. Assigning learning objectives to this column means "putting elements together to form a novel, coherent whole or make an original product" [15].

According to the three steps A), B), and C) of teaching about FSMs, our learning objectives develop step-by-step the learning content by addressing different Knowledge Dimensions as well as Cognitive Process Dimensions [6]. Each step is addressed by its related category of learning objectives. Each category covers three learning objectives - in total we identified nine learning objectives (see Fig. 1).

a) Basics of a Finite State Machine:

LO_1_A1, LO_2_B2, and LO_3_C2

b) Parallels between Finite State Machines and Stock Trading:

LO_4_C2, LO_5_D2, and LO_6_C3

c) Construction of Artificial Intelligence using Finite State Machines:

LO_7_D3, LO_8_D4, and LO_9_D5

The majority of the learning objectives is positioned on the left side of the table, namely in the columns Remember and Understand. However, with increasing use of FSTG the learning objectives grow more complex approaching other Cognitive Process as well as Knowledge Dimensions. Consequently, the learning objectives LO_5, LO_7, LO_8 and LO_9 introduce the Metacognitive Knowledge to the students.

The learning objectives of category A that cover the basics of an FSM were already discussed in [6], while the learning objectives of category B have been presented in [18]. Therefore, the following sections of this paper focus on the learning objectives of the category $\mathrm{C}$, the construction of Artificial Intelligence using FSMs. The logic of 
Paper-Automated Stock Trading - Developing the Serious Game FSTG to Teach the Topic of Finite State Machines

\begin{tabular}{|c|c|c|c|c|c|c|c|}
\hline & \multicolumn{6}{|c|}{ The Cognitive Process Dimension } \\
\hline & & $\begin{array}{c}1 . \\
\text { Remember }\end{array}$ & $\begin{array}{c}2 . \\
\text { Understand }\end{array}$ & $\begin{array}{c}3 . \\
\text { Apply }\end{array}$ & $\begin{array}{c}4 . \\
\text { Analyze }\end{array}$ & $\begin{array}{c}5 . \\
\text { Evaluate }\end{array}$ & $\begin{array}{c}6 . \\
\text { Create }\end{array}$ \\
\hline \multirow{5}{*}{ 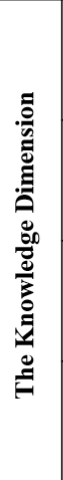 } & $\begin{array}{c}\text { A. } \\
\text { Factual } \\
\text { Knowledge }\end{array}$ & LO_1_A1 & A & & & & \\
\hline & $\begin{array}{c}\text { B. } \\
\text { Conceptual } \\
\text { Knowledge }\end{array}$ & & LO_2_B2 & & & & \\
\hline & C. & & LO_3_C2 & \multirow{2}{*}{ LO_6_C3 } & & & \\
\hline & $\begin{array}{l}\text { Procedural } \\
\text { Knowledge }\end{array}$ & & LO_4_C2 & & & & \\
\hline & \begin{tabular}{|c|} 
D. \\
Metacognitive \\
Knowledge
\end{tabular} & B & LO_5_D2 & LO_7_D3 & LO_8_D4 & LO_9_D5 & \\
\hline
\end{tabular}

Fig. 1. Classification of FSTG's learning objectives according to Bloom's Taxonomy [6]

the NPC evolves the more learning objectives are met. It is important to note, that the single evolution stages of the NPC's logic do not represent a working FSM which could be directly implemented into a software.

An FSM may either be represented by a State Diagram, a State Table or the Tuple Representation [6]. This paper relies on State Diagrams.

\subsection{Construction of Artificial Intelligence using FSMs}

By introducing the use of automation in stock trading, the complexity of the learning objectives increases. Thus, the NPC logic needs to grow to implement the required economic issues into the logic. Consequently, in order to explain Artificial Intelligence and how it can be analyzed and evaluated, the spare states and transitions which are needed for a working logic are added to the NPC's FSM. Artificial Intelligence is an omnipresent topic these days. For the implementation of the NPC of the Serious Game solution an Artificial Intelligence was developed as well. FSMs provide a suitable tool to create an intelligent logic.

The conclusive learning objectives LO_7_D3, LO_8_D4, and LO_9_D5 explain the construction of Artificial Intelligence by using FSMs and the emerging benefits and limits.

LO_7_D3: The students shall be able to apply the use of Finite State Machines for the representation of Artificial Intelligence.

In order to explain this learning objective LO_7_D3 as well as the next learning objectives, the FSM logic is extended again (see Fig. 2). All of the economic issues of the NPC logic have been implemented by now. This includes a completely performed implementation of the trading strategies presented in [6]. The FSM makes its deci- 
Paper-Automated Stock Trading - Developing the Serious Game FSTG to Teach the Topic of Finite State Machines
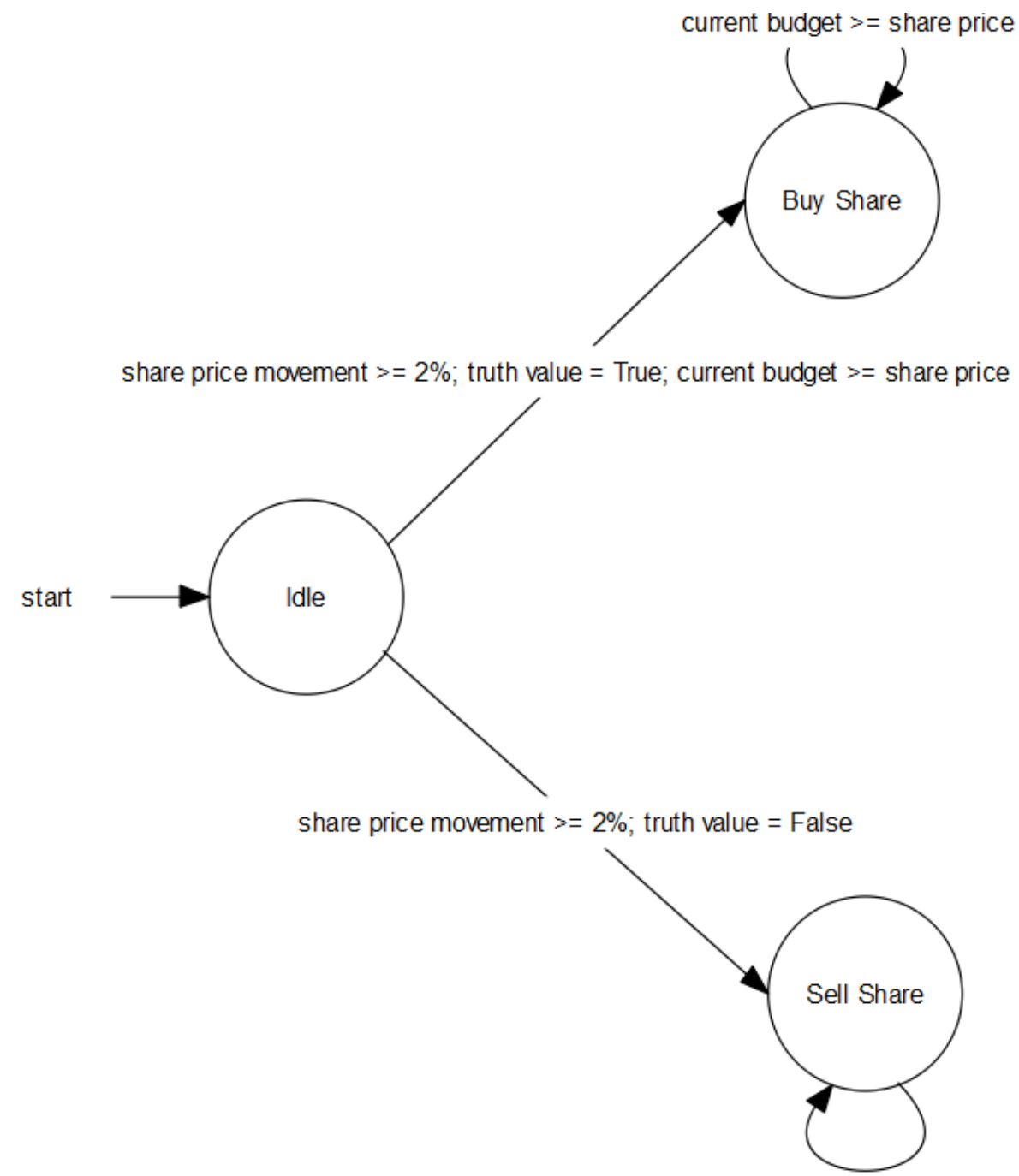

Fig. 2. Fourth evolution stage of the Non-Player Character Finite State Machine

sions dependent on a certain positive or negative share price movement. In the software, a share price delta of two percent or more between the last turn and the current turn was used for triggering an action. This delta is called share price movement in the following evolution stages. Since the two strategies are contrary, a truth value, which is randomly assigned before each round, is needed to distinguish the conditions of the transitions to the buy state and the sell state, respectively. This truth value decides whether the NPC takes the cyclical or the anti-cyclical strategy. Also, the available budget of the NPC appears the first time and is added to the condition of the transitions to the buy state. This is a crucial step to ensure a realistic trade setting. The iterative transition from the buy state to the buy state is added as well. The NPC is now 
capable of buying more than one share as long as the condition current budget $>=$ share price is fulfilled.

Although the term FSM is not familiar to most of the people, FSMs can be found everywhere in daily life. The traffic light that helps to control the traffic, the vending machine that can sell products without the help of human power, the turnstile at the entrance of attractions that can monitor the amount of people entering are just some examples. Not to forget the computer game where we interact with NPCs and fulfill goals to complete the game. There is a broad variety of definitions for Artificial Intelligence, of which none can claim to be the ultimate one [19]. Also for human intelligence there are many definitions available too [20]. Especially in the context of this paper, artificial intelligence can be seen as a synthesis of simple steps to a consistent and logical behavior similar to the one of a human. The FSM of the NPC follows this definition as well, although some parts are still missing. By using only a couple of transitions and states the intelligence of a broker has been imitated.

LO_8_D4: The students shall be able to analyze the behavior of the computercontrolled player in the game to gain advantages over it.

Once the students are introduced to the entire FSM of the NPC, they can start proving their learnings. Due to the fixed processing, this FSM can possibly be exploited by analyzing the behavior and the representation properly.

Fig. 3 shows the last evolution stage of the FSM. In this step all the missing parts to ensure a consistent and fully functional logic have been added. This includes changes on almost all conditions of the logic. The NPC shall only be allowed to sell shares, if the respective share is in stock. This is ensured via a check for a positive value of shares for both transitions leading to the sell state. In order to indicate the end of the trading process, a new state has been introduced, the Stop Trade state. This state is a final state, indicated by a double circle instead of a single one in the State Diagram.

There are two transitions leading to the Stop Trade state, one from the buy state and one from the sell state. Instead of deciding whether to stay in the respective trade state by a truth value, this decision is now done based on random values. The use of random values allows to shift the probabilities whether to continue or to stop the trading from a strict 50/50 share to a freely selectable percentage. The FSM in Fig. 3 depicts a 75 percent chance to stay in the respective trade state and a 25 percent chance to switch to the Stop Trade state. Again, with this balancing of the probabilities we strive to simulate human behavior. In fact, the probabilities are slightly differing from the ones just presented, since there is a chance that the NPC runs out of money or out of shares, respectively. This would make the switch to the Stop Trade state obligatory.

In the Serious Game solution, the complete logic used by the NPC is presented to the students. After learning the content of the previous learning objectives, and observing the behavior of the NPC in the software, the students are expected to understand the construction of the FSM of the NPC by now. It is important to note, that the presented State Diagram depicts the behavior of the NPC for one company. Thus, this logic is repeated for each of the companies available for trading. 
Paper-Automated Stock Trading - Developing the Serious Game FSTG to Teach the Topic of Finite State Machines

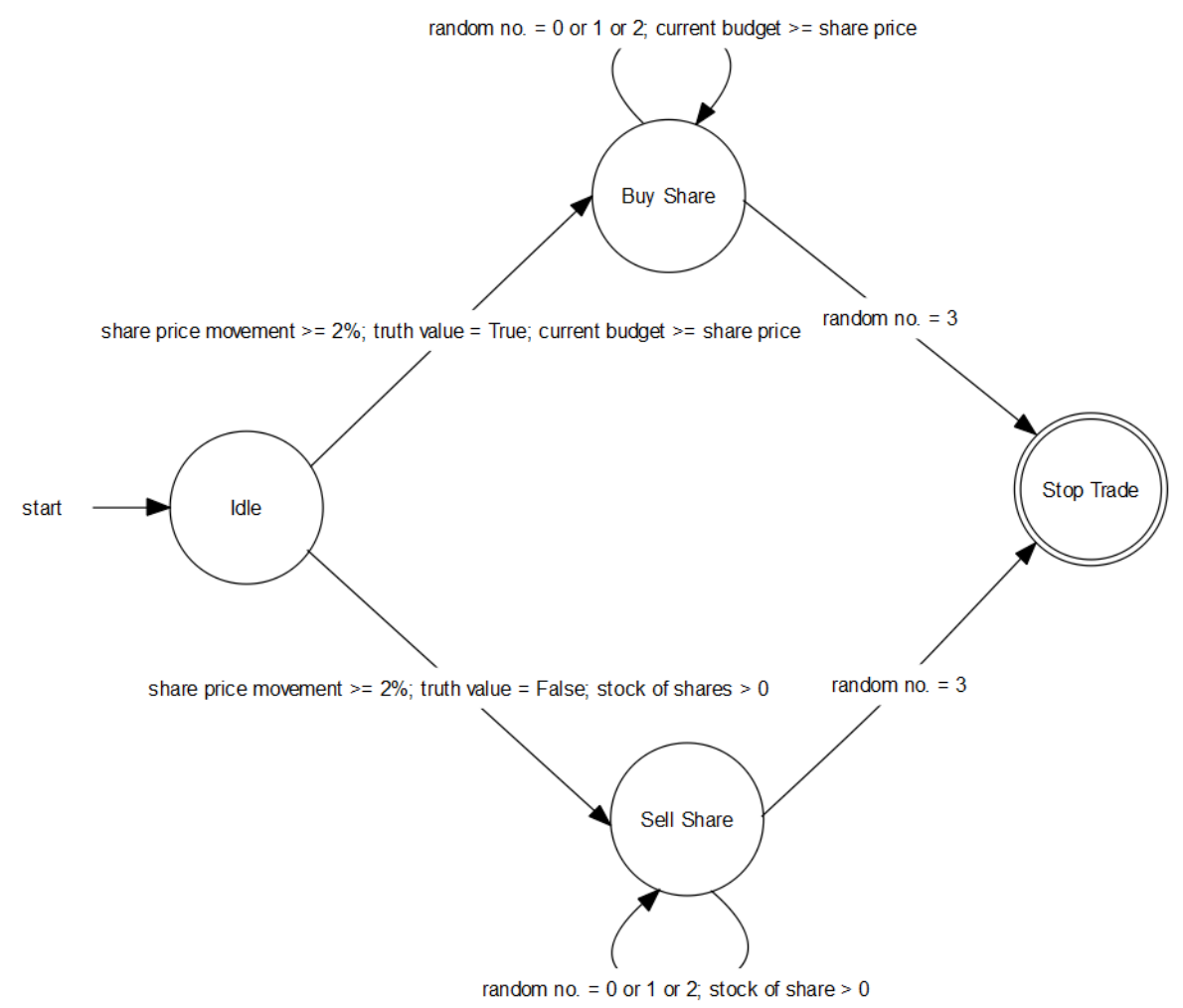

Fig. 3. Fifth evolution stage of the Non-Player Character Finite State Machine

Automated systems always follow the determined rules. This is an advantage and a disadvantage of such systems at the same time. While this behavior of automated systems makes sure that the systems are consistently and logically operating, it makes them also vulnerable for possible exploits. The students can observe which events will trigger an action of the NPC. On this way, the NPC could for example be animated to buy shares by initiating a sufficient stock price movement, since the NPC is tied to this logic and will always start an action in this case. In order to do so, it can be helpful for the student to know not only the states and conditions of the NPC FSM, but also to understand what input is used for the processing of the logic. While in the first evolution stages only the current share price was used for making decisions, more and more input is needed for the NPC behavior now. In fact, the complete FSM of the NPC takes the share prices of the last round and the current round, the player's budget, the player's stock of shares of each company as well as the randomly assigned truth value and random numbers as basis for the decision making. So, by thoroughly analyzing the behavior as well as the depicted State Diagram of the NPC the students can possibly improve their winning chances and gain advantage over the NPC.

However, it is to be noted that some seldom used transitions are not represented in the State Diagram in order to give a better overview of the logic and not to distract 
the students from the actual content. Fig. 4 shows an example of such a transition. If the share price changes less than two percent, neither the transition to the buy state nor to the sell state can be triggered. Thus, the FSM directly switches from the Idle to the Stop Trade state. Other cases are the transition from the Buy Share state to the Stop Trade state if the budget is not sufficient to buy another share, and the transition from the sell state to the Stop Trade state if the processed share is not in stock, respectively.

LO_9_D5: The students shall be able to evaluate the limits of analyzing Finite State Machines

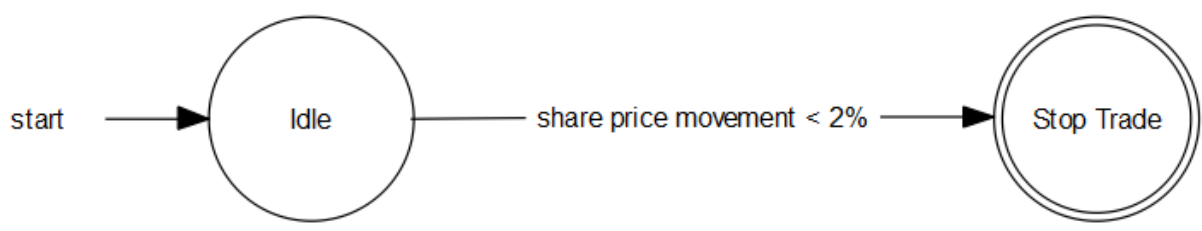

Fig. 4. Example for an excluded transition of the Non-Player Character Finite State Machine

Although the FSM of the NPC shows some possible exploits after inspecting the logic, there are limits for the analysis of FSMs. The students need to grasp these limits and to apply how to analyze and evaluate the logic of an FSM correctly. Now, the students know the behavior of the NPC in the software, which is implemented by an FSM. They also are familiar to possible ways how to analyze the logic and use it to their advantage. The next logical step is to get to know the limits of these analysis. While it is often an advantage to analyze the opponent's behavior in a game, it does not necessarily mean to be able to foresee all of the next actions of the NPC. To give an example, while the students know that the NPC will react to a certain share price movement, they cannot predict the distribution of the randomly assigned truth value and the random number. As a result, the students might be capable of estimating the next step of the NPC or analyzing all of the possible moves of the NPC. But they are not able to predict the NPC behavior completely. Summarized, implementation ways like random assigned variables help not only to improve the realism of an automated behavior, but also to keep the behavior unpredictable to a certain level. This is when the "flow" starts $[13,21]$.

\section{Application}

The Finite State Trading Game (FSTG) is a Serious Game about FSMs which encompasses all of the presented learning objectives. In this turn-based game the user strives to successfully trade shares on the market to make profit in order to win the game against an NPC, whose logic bases on an evolving FSM [6]. Table I presents a side-by-side overview of the learning objectives and the corresponding NPC's evolution stages of FSTG. 
Paper-Automated Stock Trading - Developing the Serious Game FSTG to Teach the Topic of Finite State Machines

Table 1. Learning objectives, evolution stages and test statements

\begin{tabular}{|c|c|c|c|}
\hline & Learning objective (LO) & Evolution stage (ES) & Test statement (TS) \\
\hline \multirow{3}{*}{ 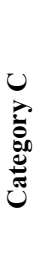 } & $\begin{array}{l}\text { LO_7_D3: The students shall be able to apply the } \\
\text { use of Finite State Machines for the representation } \\
\text { of Artificial Intelligence. }\end{array}$ & $\begin{array}{l}\text { ES4: NPC follows } \\
\text { all needed economical } \\
\text { rules }\end{array}$ & TS_8_D3 \\
\hline & $\begin{array}{l}\text { LO_ } 8 \text { D4: The students shall be able to analyze } \\
\text { the behavior of the computer-controlled player in } \\
\text { the game to gain advantages over it. }\end{array}$ & \multirow{2}{*}{$\begin{array}{c}\text { ES5: NPC } \\
\text { is fully functional } \\
\text { and acts consistently and } \\
\text { stable }\end{array}$} & TS_9_D4 \\
\hline & $\begin{array}{l}\text { LO_9_D5: The students shall be able to evaluate } \\
\text { the limits of analyzing Finite State Machines. }\end{array}$ & & TS_10_D5 \\
\hline
\end{tabular}

In order to verify the FSTG approach the software was tested as part of the Pupils' Academy of Serious Gaming for $11^{\text {th }}$ grade UVS students [22]. The details of the test lesson itself are fully described in [6].

According to [6] we created a questionnaire to quantify the test results. The questionnaire relies on a five point Likert scale [23]: 1 - Strongly Disagree, 2 - Disagree, 3 - Neither Agree nor Disagree, 4 - Agree and 5 - Strongly Agree. The participants self-assessed their knowledge before the start of the teaching unit in a pre-test and after the end of the teaching unit in a post-test.

The classification of the test statements corresponds to the classification of the learning objectives in Bloom's Taxonomy (see Fig. 1). The statements are discussed shortly for the category C - for A see [6], while for B see [18].

TS_8_D3: I am familiar with the term "Artificial Intelligence" and its correlation with Finite State Machines.

Artificial Intelligence is a term, which is used more and more often in daily life. But the connection to FSMs is way less known to many people. The comparison of the answers to this statement in the pre-test and the post-test verifies whether the students were able to understand one of the main contents of the software.

TS_9_D4: I understand why "Artificial Intelligence" is an accumulation and succession of simple steps, actions and conditions.

A positive answer to this statement shows that the student is familiar with the synthesis of single steps into a functional logic. Particularly after using the software high scoring answers could show that the students can apply the construction of the logic in the software at the minimum.

TS_10_D5: I understand why automated processes are potentially vulnerable for exploiting due to their rigidly defined logic.

This statement is expected to be answered not very positively by the students in the pre-test due to the detailed level of the statement. Nevertheless, any positive answers to the statement in the post-test prove that the students were able to connect the core ideas of the presented content of the paper and thus to understand potential weaknesses of the presented automated system. 


\section{$4 \quad$ Results}

The students filled the same questionnaire two times on separate documents. This means that they assessed their knowledge twice: before and after they used FSTG [6]. This paper focuses on the test statements TS 8 D3 to TS 10 D5.

\section{A. Evaluation of the Pre-Test}

The answers to the statements TS_8_C3, and TS_9_D4 show that the students were more familiar to the areas related to the topic Artificial Intelligence. But the answers were lopsided to the negative side with only about $18.33 \%$ of the answers being on the positive side. In the statement TS_10_D5 the number of positive answers peaked in $50 \%$ share of the overall answers. These results being higher than the ones in the previous statements, which were tested in category A [6] and category B [18], was probably the result of omitting the term Finite State Machine. The use of more daily terms appeared to result in more positive answers by the students. Also the test statement tested a more general knowledge about automation and thus approached a bigger scope of the topic. In test statement TS_8_D3 the students appear to have concentrated on being familiar with Artificial Intelligence, while ignoring the second part of the test statement.

\section{B. Comparison of the Pre-Test and the Post-Test}

In order to allow a better overview for the two test rounds, the results of the pretest and the post-test need to be compared. Fig. 5 shows the five (dis)agreement levels of the Likert Scale as well as depicts the answers to the last three test statements, which are assigned to category $\mathrm{C}$. The students assessed their knowledge far more positive in the post-test than in the pre-test. In particular, test statement TS_8_D3, "I am familiar with the term 'Artificial Intelligence' and its correlation with Finite State Machines" shows a significant improvement. Before the students used the software, none of them rated this test statement as 5 - Strongly Agree and only $10 \%$ chose $4-$ Agree. After they tried the Serious Game solution, the target group reported a considerable improvement: 55\% selected 5-Strongly Agree and 35\% 4-Agree.

\subsection{Findings}

The results of the post-test seem to verify the success of our FSTG approach to ITbased learning of FSMs. The evaluation statements with less or no progress show, which parts of the software need some rework.

As a qualitative result the Serious Game solution succeeded to keep the students motivated during the lesson without further incentives. On the quantitative side, major progress on every statement can be shown [6]. Only $0.05 \%$ of all answers of the posttest were negative. This is a remarkable fact, when compared to overall $73.50 \%$ negative answers in the pre-test. Hence the number of positive answers shifted from $16.50 \%$ in the pre-test to $89.00 \%$ positive answers in the post-test. However, a shift of the answered options to the positive side had to be expected due to the fact that most statements are directly addressed in the software. Nevertheless, this test is a 


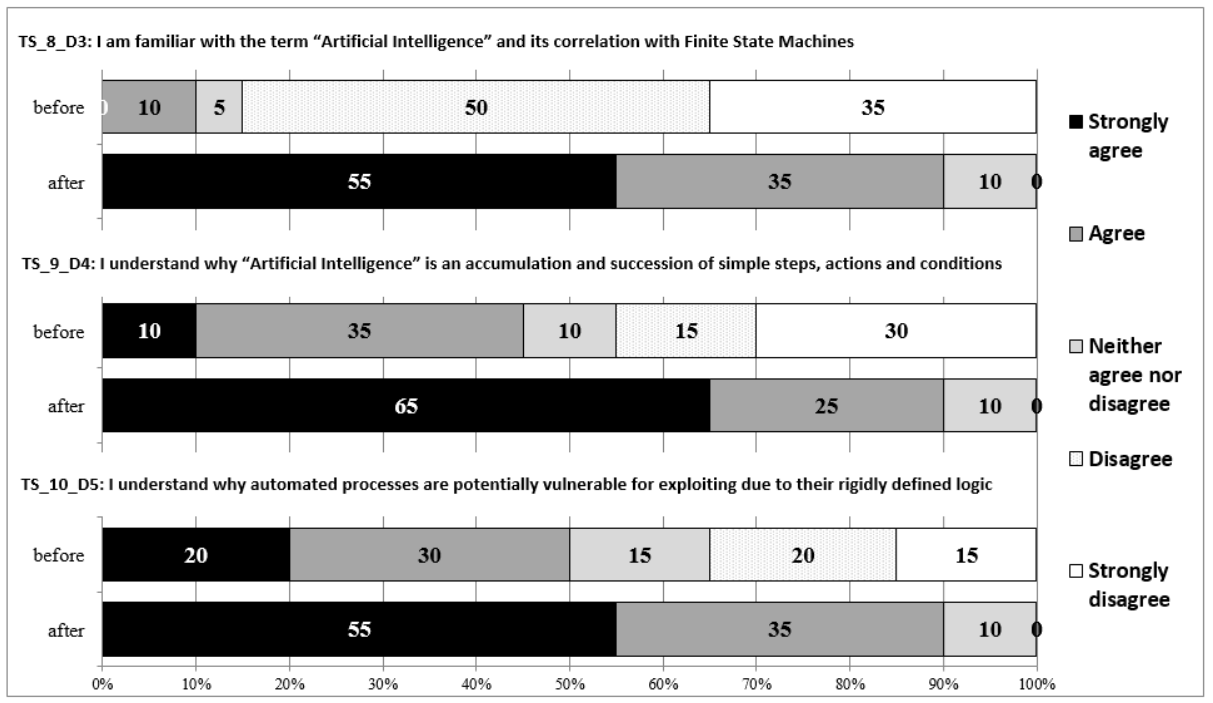

Fig. 5. Results of the answers to the test statements of Category $\mathrm{C}$ in the pre-test and the posttest

success, since big progress was recorded in all test statements. The positive results in category $\mathrm{C}$ of the post-test prove that the students understood the application of FSMs and their use to construct Artificial Intelligence. Although showing some foreknowledge in the pre-test, the students seemed to be way more comfortable with their knowledge about the use of FSMs - as well as the topic Artificial Intelligence in general - after using FSTG.

\section{Conclusion}

The upper vocational schools (UVS) are an important part of the Bavarian school system in Germany. The use of Finite State Machines (FSM) allows to cover essential modules of the UVS, like systems and processes or the capstone project, due to the simple scalability and the widespread application Fields of FSMs. By combining these advantages with the merits Self-Regulated Personalized Learning offers, an ITbased Serious Game seems to be a favorable methodology to teach about the concept of FSMs.

Consequently, the purpose of this research was to design, create, and present an ITbased learning solution in the form of a Serious Game to teach the topic of FSMs to UVS students in Bavaria by using stock trading as an example.

In order to fulfill this research goal, we created, classified and verified nine learning objectives for teaching about FSMs by applying the Taxonomy of Bloom [15]. These learning objectives were allocated into the three categories, which covered the basics of FSMs, the parallels between FSMs and stock trading as well as the application of FSMs and their use to create Artificial Intelligence. This research addresses 
Category C, covering how Finite State Machines can be used to construct Artificial Intelligence, before discussing benefits and limitations of this method.

In the following step, the learning objectives were integrated and implemented into the Serious Game "The Finite State Trading Game" (FSTG), a turn-based game in which the user strives to trade shares efficiently to beat the non-player character (NPC) opponent by making higher profits. The game is structured in several levels, all of them offering different stages of complexity. The NPC follows a FSM logic to make its trading decisions. By using graphical representations of this FSM logic, the user gets explained important knowledge about the characteristics as well as the behavior of the computer-controlled NPC during the course of the game.

The next step - the practical test of the Serious Game - was performed in a lesson as part of the Pupils' Academy [24] at the Technical University of Munich. Students of the UVS Staatliche Fachober- und Berufsoberschule Technik München selfassessed their knowledge about Finite State Machines just before and right after playing FSTG in order to record any progress they have made on the topic. The test statements for this test scenario were created by using the five-point scale of Likert [23] and once again the Taxonomy of Bloom. On this way we were able to verify that the test statements matched the learning objectives of FSTG.

Based on the following evaluation of the test sheets, the test on the target group can be rated as a success. While in the pre-test the majority of the given answers (73.5 percent) were negative, only 0.05 percent of the given answers in the post-test were rated as negative answers. Thus, a significant shift to the positive side of the answers can be recorded for each of the test statements when comparing the answers of the pre-test with the answers of the post-test.

Due to the successful test setting, the Serious Game seems to be ready for usage and testing in a larger scale and in different classrooms. The results of the evaluation show, that the learning objectives fit to the requirements to teach the FSM concept and that they are suitable for the target group consisting of UVS students. Since the UVS and the German Gymnasium clearly show existing parallels, a test setting in a Gymnasium class could provide interesting insights. Former research [22] has shown positive impact of playing a Serious Game on the results in the final exam, the German Abitur. Thus, we want to focus with our research on this aspect. Additionally, by increasing the teaching content and the functionalities, FSTG could be developed to a stand-alone way of teaching instead of the current situation as a supplement to the traditional way of teaching.

FSTG shows opportunities to be vertically expanded as well. After beating all the levels of FSTG the users could get the option to create an own FSM Models themselves in order to play and win against the created logic. On this way the students can prove their learnings by applying their learned knowledge. This subsequent process of learning and applying knowledge can help the student to intensify their know-how. Additionally, this method would verify the construction of the Serious Game and thus this research. 


\section{Acknowledgment}

The authors would like to thank the anonymous reviewers for their very helpful suggestions and comments. Their inputs have significantly helped to improve this publication. Furthermore, we would like to express special thanks to the upper vocational schools at Bavaria represented by Günter Liebl, Werner Maul, and Konrad Maurer.

\section{$7 \quad$ References}

[1] Entertainment Software Associate ESA. Essential Facts about the Computer and Video Game Industry [Online]. Available: http://www.isfe.eu/sites/isfe.eu/files/attachments/esa ef 2011.pdf

[2] P. Black. Finite State Machine [Online]. Available: http://xlinux.nist.gov/dads/HTML/ finiteStateMachine.html

[3] Bayerisches Staatsministerium für Unterricht und Kultus, "Lehrpläne für die Fachoberschule und Berufsoberschule Ausbildungsrichtungen Technik, Agrarwirtschaft, Gestaltung Unterrichtsfach: Technologie/Informatik Ausbildungsrichtung Wirtschaft, Verwaltung und Rechtspflege Unterrichtsfach: Technologie Jahrgangsstufen 11 bis 13 ", ed, 2006.

[4] Technical University of Munich. (2012, May 24). Bachelor's Program Informatics: Curriculum and Courses. Available: http://www.in.tum.de/en/current-students/bachelorsprograms/informatics/curriculum-and-courses.html

[5] T. Nipkow. (2011, May 24). Introduction to Theory of Computation. Available: https://campus.tum.de/tumonline/WBMODHB.wbShowMHBReadOnly?pKnotenNr=4540 $45 \& \mathrm{pOrgNr}=14189$

[6] M. Utesch, A. Hauer, R. Heininger, and H. Krcmar, "An IT-based Learning Approach about Finite State Machines Using the Example of Stock Trading," in Interactive Collaborative Learning (ICL) 2016, Belfast, UK, 2016.

[7] P. Adamczyk, "The Anthology of the Finite State Machine Design Patterns," in The 10th Conference on Pattern Languages of Programs, 2003.

[8] Docebo. E-Learning Market Trends \& Forecast 2014 - 2016 Report [Online]. Available: https://www.docebo.com/landing/contactform/elearning-market-trends-and-forecast-20142016-docebo-report.pdf

[9] B. J. Zimmerman, "Becoming a Self-Regulated Learner: an Overview," Theory into practice, vol. 41, pp. 64-70, 2002. https://doi.org/10.1207/s15430421tip4102 2

[10] M. Utesch, R. Heininger, and H. Krcmar, "Strengthening Study Skills by using ERPsim as a new Tool within the Pupils' Academy of Serious Gaming," in 2016 IEEE Global Engineering Education Conference (EDUCON), 2016, pp. 592-601. https://doi.org/10.1109/EDUCON.2016.7474611

[11] M. Andergassen, G. Ernst, V. Guerra, F. Mödritscher, M. Moser, G. Neumann, et al., "The Evolution of e-Learning Platforms from Content to Activity Based Learning: The Case of Learn@WU," in Interactive Collaborative Learning (ICL), 2015 International Conference on, 2015, pp. 779-784. https://doi.org/10.1109/icl.2015.7318127

[12] Initiative D21. Bildungsstudie: Digitale Medien in der Schule [Online]. Available: http://www.initiatived21.de/wp-content/uploads/2011/05/NOA_Bildungsstudie_140211 .pdf 
[13] R. Heininger, M. Utesch, and H. Krcmar, "Schülerakademie Serious Gaming mit ERPsim zur Förderung der Studierfähigkeit," in Multikonferenz Wirtschaftsinformatik (MKWI) 2016, Ilmenau, Germany, 2016.

[14] B. S. Bloom, Taxonomy of Educational Objectives. Vol. 1: Cognitive Domain: New York: McKay, 1956.

[15] D. R. Krathwohl, "A Revision of Bloom's Taxonomy: an Overview," Theory into practice, vol. 41, pp. 212-218, 2002. https://doi.org/10.1207/s15430421tip4104_2

[16] L. Yuan and G. Qu, "Information Hiding in Finite State Machine," in Information Hiding: 6th International Workshop, IH 2004, Toronto, Canada, May 23-25, 2004, Revised Selected Papers, J. Fridrich, Ed., ed Berlin, Heidelberg: Springer Berlin Heidelberg, 2005, pp. 340-354. https://doi.org/10.1007/978-3-540-30114-1 24

[17] L. W. Anderson and D. R. Krathwohl, "A Taxonomy for Learning, Teaching, and Assessing: a Revision of Bloom's Taxonomy of Educational Objectives," ed: Allyn and Bacon, 2001.

[18] M. Utesch, A. Hauer, R. Heininger, and H. Krcmar, "The Finite State Trading Game: Developing an IT-based Learning Solution to teach the Application of Finite State Machines in a Stock Trading Scenario," in 2017 14th International Conference on Remote Engineering and Virtual Instrumentation (REV), New York, USA, 2017, in press.

[19] S. Russell and P. Norvig, Artificial Intelligence: a Modern Approach, 2 ed.: Prentice Hall, 2002.

[20] S. Legg and M. Hutter, "Universal Intelligence: a Definition of Machine Intelligence," Minds and Machines, vol. 17, pp. 391-444, 2007. https://doi.org/10.1007/s11023-0079079-x

[21] M. Csikszentmihalyi, Beyond Boredom and Anxiety: Jossey-Bass Publishers, 1975.

[22] M. Utesch, R. Heininger, and H. Krcmar, "The Pupils' Academy of Serious Gaming: Strengthening study skills with ERPsim," in 2016 13th International Conference on Remote Engineering and Virtual Instrumentation (REV), 2016, pp. 93-102. https://doi.org/10.1109/rev.2016.7444446

[23] R. Likert, "A Technique for the Measurement of Attitudes," Archives of psychology, 1932.

[24] M. C. Utesch, "Five years of the Pupils' Academy of Serious Gaming: Enhancing the ability to study," in 2015 IEEE Global Engineering Education Conference (EDUCON), 2015, pp. 189-198. https://doi.org/10.1109/EDUCON.2015.7095970

\section{Authors}

Dr. M. C. Utesch is with the upper vocational school 'Staatliche Fachober- und Berufsoberschule Technik', Bergsonstraße 109, 81245 Munich and the Technical University of Munich (TUM), Department of Informatics, Chair for Information Systems, Boltzmannstr. 3, D-85748 Garching, Germany (e-mail: utesch@in.tum.de).He is a member of the Executive Committee of IGIP. He was a member of the Core Advisory Board of the EU Sixth Framework Programme/iclass. His main interests include IT-based learning, games engineering, and in particular the enhancement of the study skills at the interface between school and university.

A. Hauer is a masters student at the Technical University of Munich (TUM), Department of Informatics, Chair for Information Systems, Boltzmannstr. 3, D-85748 Garching, Germany (e-mail: ga67bik@mytum.de). His main interests include Serious Games as well as software and app development. 
Robert Heininger is a research associate at the Technical University of Munich (TUM), Department of Informatics, Chair for Information Systems, Boltzmannstr. 3, D-85748 Garching, Germany (e-mail: robert.heininger@in.tum.de). He is responsible for the school programs at the Department of Informatics. His research activities focus on IT service management, service automation, cloud computing, and IT-based learning.

Prof. Dr. Helmut Krcmar is a full professor of Information Systems at the Technical University of Munich (TUM), Department of Informatics, and holder of the Chair for Information Systems since 2002, Boltzmannstr. 3, D-85748 Garching, Germany (e-mail: krcmar@in.tum.de). He worked as a Post Doctoral Fellow at the IBM Los Angeles Scientific Center, as Assistant Professor of Information Systems at the Leonard Stern School of Business, NYU, and at Baruch College, CUNY. From 1987 to 2002 he was Chair for Information Systems, Hohenheim University, Stuttgart. He has been actively involved in numerous Association of Information Systems (AIS) activities, e.g. as President of the AIS, is an AIS fellow and is active in many other functions. His research interests include digitization, Information and Knowledge Management, IT-enabled Value Webs, Service Management, Computer Supported Cooperative Work, and Information Systems in Health Care and eGovernment.

This article is an extended and modified version of a paper presented at the International Conference on Interactive Collaborative Learning (ICL2016), held September 2016, in Belfast, UK. Article submitted 14 December 2016. Published as resubmitted by the authors 26 January 2017. 